\title{
Wolbachia, Sodalis and trypanosome co-infections in natural populations of Glossina austeni and Glossina pallidipes
}

Florence N Wamwiri ${ }^{1 *}$, Uzma Alam², Paul C Thande ${ }^{1}$, Emre Aksoy², Raphael M Ngure ${ }^{3}$, Serap Aksoy², Johnson O Ouma ${ }^{1}$ and Grace A Murilla ${ }^{1}$

\begin{abstract}
Background: Tsetse flies harbor at least three bacterial symbionts: Wigglesworthia glossinidia, Wolbachia pipientis and Sodalis glossinidius. Wigglesworthia and Sodalis reside in the gut in close association with trypanosomes and may influence establishment and development of midgut parasite infections. Wolbachia has been shown to induce reproductive effects in infected tsetse. This study was conducted to determine the prevalence of these endosymbionts in natural populations of G. austeni and G. pallidipes and to assess the degree of concurrent infections with trypanosomes.
\end{abstract}

Methods: Fly samples analyzed originated from Kenyan coastal forests (trapped in 2009-2011) and South African G. austeni collected in 2008. The age structure was estimated by standard methods. G. austeni $(n=298)$ and G. pallidipes $(\mathrm{n}=302)$ were analyzed for infection with Wolbachia and Sodalis using PCR. Trypanosome infection was determined either by microscopic examination of dissected organs or by PCR amplification.

Results: Overall we observed that G. pallidipes females had a longer lifespan (70 d) than G. austeni (54 d) in natural populations. Wolbachia infections were present in all G. austeni flies analysed, while in contrast, this symbiont was absent from G. pallidipes. The density of Wolbachia infections in the Kenyan G. austeni population was higher than that observed in South African flies. The infection prevalence of Sodalis ranged from 3.7\% in G. austeni to about $16 \%$ in G. pallidipes. Microscopic examination of midguts revealed an overall trypanosome infection prevalence of $6 \%(n=235)$ and $5 \%(n=552)$, while evaluation with ITS1 primers indicated a prevalence of about 13\% $(n=296)$ and $10 \%(n=302)$ in G. austeni and G. pallidipes, respectively. The majority of infections (46\%) were with T. congolense. Co-infection with all three organisms was observed at 1\% and 3.3\% in G. austeni and G. pallidipes, respectively. Eleven out of the thirteen (85\%) co-infected flies harboured T. congolense and T. simiae parasites. While the association between trypanosomes and Sodalis infection was statistically significant in G. pallidipes $(P=0.0127)$, the number of co-infected flies was too few for a definite conclusion.

Conclusions: The tsetse populations analyzed differed in the prevalence of symbionts, despite being sympatric and therefore exposed to identical environmental factors. The density of infections with Wolbachia also differed between G. austeni populations. There were too few natural co-infections detected with the Sodalis and trypanosomes to suggest extensive inter-relations between these infections in natural populations. We discuss these findings in the context of potential symbiont-mediated control interventions to reduce parasite infections and/or fly populations.

Keywords: Glossina, Wolbachia, Sodalis, Trypanosomes, Co-infection, Shimba Hills, Kenya

\footnotetext{
* Correspondence: F.wamwiri@gmail.com

${ }^{1}$ KARI-Trypanosomiasis Research Centre, P.O. Box 362-00902, Kikuyu, Kenya

Full list of author information is available at the end of the article
} 


\section{Background}

Tsetse and trypanosomiasis continue to be a constraint to agricultural development in tsetse-infested regions of sub-Saharan Africa. Tsetse flies are the sole insect vectors responsible for transmission of both human and animal trypanosomiasis. The methods to control disease in the mammalian host are very limited. Traditional methods of vector control, such as traps and targets, have time and again proven unsustainable [1], turning the attention of scientists to the development of alternative methods, including genetic-based control such as the Sterile Insect Technique (SIT) and Incompatible Insect Technique (IIT).

All tsetse species are susceptible to trypanosome infections. However a differential vectorial capacity closely tied to differences in refractoriness to trypanosome infections are observed in tsetse [2,3]. In general, the palpalis group of flies tends to be poorer vectors of trypanosomes than the morsitans group. Although, the fly immune system is a key factor in this refractoriness [4], the natural symbiont-tsetse-trypanosome tripartite relationship has also been suggested to influence tsetse's refractoriness. The presence of gut endosymbionts in Glossina and other obligate feeders has long been established [5]. All tsetse flies rely on the obligate primary symbiont Wigglesworthia glossinidia for vital physiological functions, including maintenance of fecundity and immunity [6,7]. In addition, some natural tsetse populations harbor the symbionts Wolbachia and Sodalis. While Wolbachia resides in reproductive organs, Sodalis is found in the tsetse midgut, which is also the site of trypanosome maturation. Thus, the interactions between symbionts and trypanosomes may provide an avenue for disease control.

The prevalence of symbiotic infections in different tsetse species and natural populations vary and indeed some individuals carry none except for the primary symbiont Wigglesworthia [8]. Recent work carried out with G. $f$. fuscipes from various populations in Uganda did not detect any Sodalis infections, but low density infections with Wolbachia were reported [9]. In the G. p. palpalis populations in West Africa, a low but statistically significant association was found between the presence of Sodalis and trypanosome infections [10]. However, the role of specific Sodalis genotypes in influencing susceptibility of the flies to infection by trypanosomes remains to be determined [11]. It appears that the infection prevalence of trypanosomes in natural populations may be influenced by the presence of different symbiotic microfauna the flies carry (reviewed by Weiss et al. [12,13]).

It has also been postulated that manipulation of the tsetse-endosymbiont interactions could present the next avenue to disease and vector control. Paratransgenic approaches, which genetically modify the symbiont in the vector, have proved successful in reducing the capacity of triatomine bugs to transmit the T. cruzi parasite
[14]. The viability of this approach has also been demonstrated in sandflies for the control of Leishmania [15], and in mosquitoes for malaria control [16-18]. The IIT method is based on the cytoplasmic incompatibility (CI) phenotype expressed by some strains of Wolbachia. CI expression results in embryonic lethality when Wolbachia infected males mate with un-infected females (referred to as uni-directional CI). Bi-directional CI is expressed when individuals with different incompatible strains of Wolbachia mate. The CI effect of Wolbachia infections has been exploited in semi-field trials to control the mosquito Culex pipiens [19], and the medfly Ceratitis capitata [20]. Recently, CI was experimentally demonstrated to be also expressed in G. m. morsitans [21]. In addition to CI, Wolbachia infections have been shown to confer additional host-fitness effects, which can be applied for disease or vector control. Some strains of Wolbachia have been shown to decrease the lifespan of their hosts [22,23], or decrease the susceptibility of their host to transmit parasitic [24] and viral infections [25]. Therefore, it is clear that novel alternative vector control methods based on use of microfauna exist and some have passed the proof of concept, being at field-testing stage [26]. For successful translation of laboratory results in actual field applications, an in-depth understanding of the prevailing natural field dynamics is required. Data on natural infection frequency and information on the circulating symbiont strain types is critical in order to evaluate the potential use of endosymbionts to modify insect vector population traits [27].

There is limited information available on Wolbachia and Sodalis infections in Kenyan tsetse populations, often describing a single collection $[8,28]$. The objective of the present study was to perform a temporal analysis for infection prevalence of the symbionts Wolbachia and Sodalis in the coastal tsetse belt of Kenya where two species, G. austeni and G. pallidipes exist in sympatry. We also compared the Kenyan G. austeni populations to two other populations obtained from South Africa. We investigated the age structure of the two populations as well as trypanosome infection prevalence over three years in order to detect temporal variations. The over-arching aim of this study was to build the foundation on tsetse's microbial partners, which can be harnessed to develop genetic based methods to reduce trypanosome infections.

\section{Methods}

Study area

Tsetse flies were trapped from two national reserves in the coastal region of Kenya, namely Shimba Hills National Reserve (SHNR) in Kwale District and the ArabukoSokoke National Reserve (ASNR) in Malindi District between 2009-2011 (Figure 1). Both areas are located 


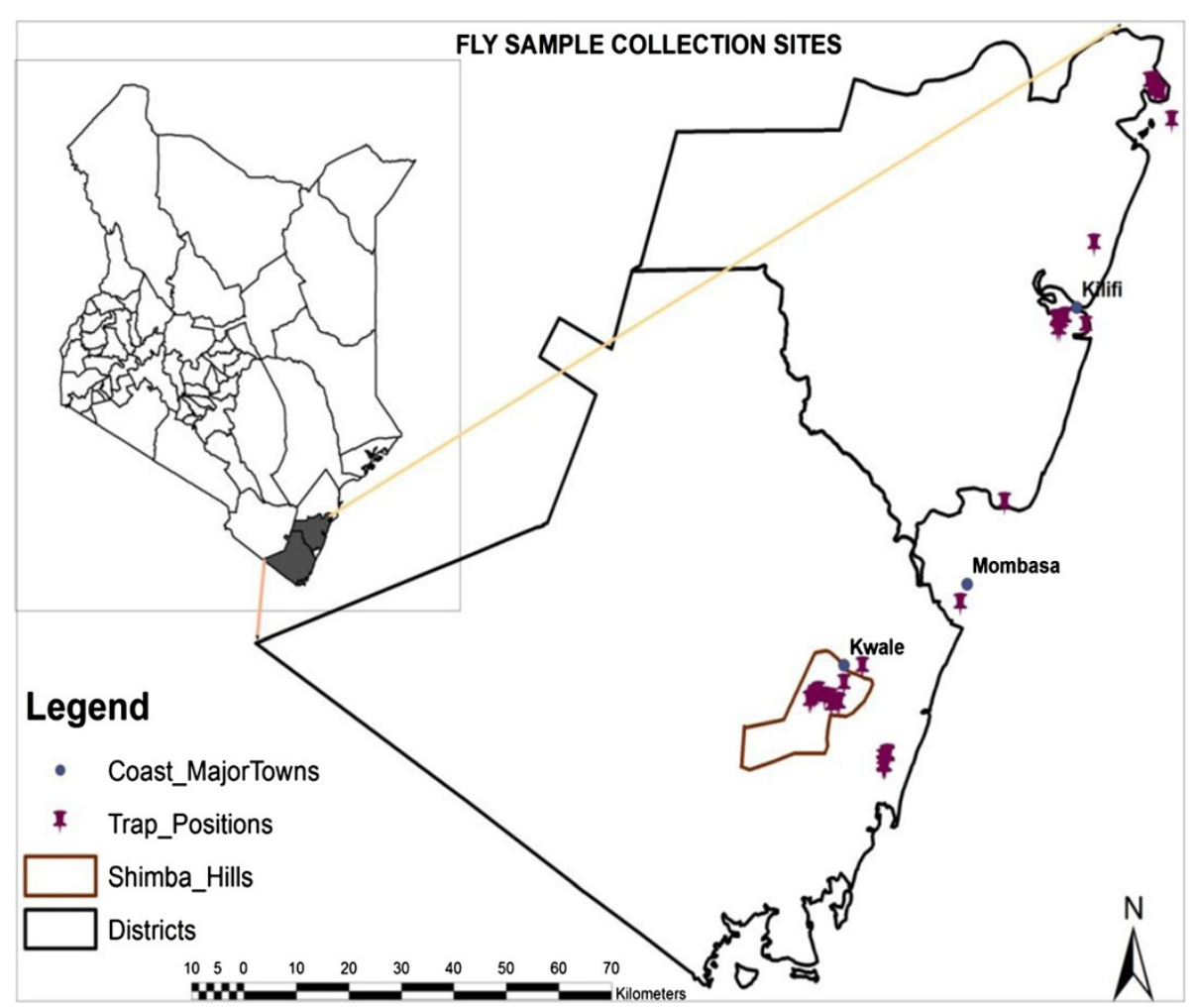

Figure 1 Fly sample collection sites in Shimba Hills, Kwale and Arabuko-Sokoke, Kilifi.

in the coastal lowlands agro-ecological zones $2-4$ which are characterized by sub-humid conditions. SHNR lies between latitude $4^{\circ} 20^{\prime} \mathrm{S}$ and longitude $39^{\circ} 31^{\prime} \mathrm{E}$, at an altitude of $120-450 \mathrm{~m}$ and covers an area of about $250 \mathrm{~km}^{2}$. The area has temperatures ranging between $24-36^{\circ} \mathrm{C}$ and rainfall averages $900-1200 \mathrm{~mm}$ per annum, falling in the short rains of October-December and the long rains of March-June. A wide range of wild animals can be found here including elephants, buffalo, antelope and waterbuck. The ASNR is located at latitude $3^{\circ} 16^{\prime} \mathrm{S}$ and longitude $39^{\circ} 49^{\prime} \mathrm{E}$. The park itself covers an area of about $6 \mathrm{~km}^{2}$ and is part of the larger Arabuko-Sokoke forest, the largest remaining fragment of coastal forest in East Africa. The forest contains three forest types namely mixed forest, Brachystegia and Cynometra tree species, each of which protects different communities of plants and animals. Like the SHNR, a wide variety of wild game is present including mongoose, duikers, forest elephants, African civets, baboons and Vervet monkeys. There are three species of tsetse flies found in the study area, namely G. pallidipes, G. austeni and G. brevipalpis.

\section{Fly collections}

Glossina austeni and G. pallidipes were trapped using either standard biconical trap [29], the Ngu2G trap [30] or the sticky mono-panel trap [31] whose lower half was coated with a thin layer of Temoocid $^{\bullet}$ adhesive (Kollant SPA, Italy). The traps were baited with a plastic sachet containing $1 \mathrm{ml}$ each of 4-methylphenol, 3-n-propyl phenol and 1-octen-3-ol. In addition, a soda bottle containing acetone was placed next to the trap. All the odours were dispensed by placing them on the windward side [32]. The traps were left in place for between 6-24 hours depending on the tsetse density in the particular area. All trap sites were geo-referenced using Garmin GPSmap76 (Garmin Corporation, Olathe, KS, USA). During the study period, all fly collections in Kenya were conducted during the months of October-November, coinciding with the short rainy season. On collection, fly catches from each trap were sorted by species and a proportion of them were preserved in $50 \mathrm{ml}$ Falcon tubes containing absolute ethanol while the remainder were dissected for parasite detection. Records of total catches, sex, date and area of collection were maintained for cross-referencing purposes. A comparative sample of $G$. austeni $(n=74)$ collected from south of Lake St. Lucia, South Africa (LSLSA) and from the northern part of the lake, specifically Mbazwana, Muzi, Phinda and Tembe ("northern") was also analyzed.

\section{DNA extraction}

Upon removal from ethanol storage, individual flies were blotted dry on a paper towel and air-dried overnight at room temperature. Total genomic DNA was extracted 
from individual whole fly samples (minus legs and wings) or individual fly sections/organs (head, thorax, abdomen, legs) using either the DNeasy ${ }^{\bullet}$ Blood and Tissue Kit (Qiagen Sciences, MD, USA) according to the manufacturer's instructions or by using the salting out protocol [33]. For G. austeni 155 and 141 samples were extracted by commercial kit and salt precipitation respectively, whereas for G. pallidipes these were 104 and 197 samples respectively. For the latter procedure, flies were crushed in $300 \mu \mathrm{l}$ TNES buffer $(50 \mathrm{mM}$ Tris $\mathrm{pH}$ 7.5, $400 \mathrm{mM} \mathrm{NaCl}, 20 \mathrm{mM}$ EDTA, 0.5\% SDS) and incubated for 3 hours at $56^{\circ} \mathrm{C}$ in the presence of proteinase $\mathrm{K}$ (20 $\mathrm{mg} / \mathrm{ml}$ ). $5 \mathrm{M} \mathrm{NaCl}$ was then added to the solution and centrifuged to precipitate the proteins. The supernatant was removed and cold ethanol added to it. A further centrifugation step to sediment the DNA was carried out followed by an additional wash in ethanol. The final DNA product was eluted in 30-50 $\mu \mathrm{l}$ of TE buffer (10 mM Tris- $\mathrm{HCl} \mathrm{pH} 7.5,1 \mathrm{mM}$ EDTA) and stored at $-20^{\circ} \mathrm{C}$ until further processing.

\section{Wolbachia and Sodalis screening}

The presence of Wolbachia was determined by amplification of the Wolbachia surface protein (wsp) gene using the primers $81 \mathrm{~F} / 691 \mathrm{R}[34,35]$, which produced a fragment of $600 \mathrm{bp}$ and confirmed by a Wolbachia-specific 16S rRNA assay [36]. The presence of Sodalis was determined using the primers GPO1F/GPO1R [37]. PCR assays were performed using $1 \mu \mathrm{l}$ of template DNA and standard conditions [9]. For each assay, a negative control (no DNA) as well as a positive control using DNA isolated from Sodalis morsitans was included. The quality of sample DNA was verified using insect-specific $12 \mathrm{~S}$ mitochondrial markers [38]. After completion of the PCR run, $10 \mu \mathrm{l}$ of the amplification product was analyzed by electrophoresis in TAE buffer on a $1-2 \%$ agarose gel together with a 100 bp DNA ladder size standard (Invitrogen, Carlsbad, CA, USA) and visualized using ethidium bromide.

\section{Determination of trypanosome infection in flies}

In the field, where possible, a small proportion of the captured flies were dissected in PBS using the method of Lloyd and Johnson [39] and examined microscopically for presence of trypanosomes. Circulating trypanosome species were identified based on their location in the fly, such that infections in the proboscis only were categorized as T. vivax, infections in both proboscis and midgut as $T$. congolense and infections in proboscis, midgut and salivary glands as T. brucei spp. Infections found in the midgut only were identified as immature infections [39]. Trypanosome infection was also detected by PCR using universal primers ITS1 CF and ITS1 BR [40]. Following DNA extraction, PCR reaction was performed in $20 \mu \mathrm{l}$ reactions for 35 cycles at an annealing temperature of $58^{\circ} \mathrm{C}$. For each PCR run, DNA from an uninfected colony fly and from known trypanosome stocks were included as negative and positive controls, respectively. Electrophoresis and visualization was carried out as described above. Identification of the infecting trypanosome(s) was based on the product size as described by Njiru et al. [40].

\section{Age structure of the different populations}

Fly age was estimated using representative samples collected over three years in SHNR where both G. austeni and G. pallidipes were present. Ageing was performed using wing fray analysis for males and ovarian ageing for females. The wings from 100 randomly selected male flies of both species were scored based on the degree of tearing on the trailing edge of the wing [41]. An additional subset of females was used to estimate the age of the population using the ovarian ageing technique [42].

\section{Results}

\section{Tsetse fly density}

A total of 6,384 G. pallidipes, 1,030 G. austeni and 504 G. brevipalpis were captured in 226 trap-days during the study period. Average trap catches varied with location, fly species and trap design. As an illustration, the sticky monopanel traps which were deployed 66 trap-days only in the first year of study captured 2.8 flies/trap/day (FTD) of G. austeni as compared to 0.9 and 0.5 FTD captured by the biconical and NGU traps respectively. However, considering all trap catches irrespective of trap type the FTDs over the three-year period was 24, 4.1 and 1.9 flies for G. pallidipes, G. austeni and G. brevipalpis respectively. All areas recorded lower FTDs in the year 2010 as compared to 2009 and 2011. Overall, the highest FTDs were recorded for G. pallidipes in SHNR with densities in excess of 60 FTD. No G. pallidipes flies were captured in the ASNR.

\section{Age structure of G. austeni and G. pallidipes}

Our analysis revealed a relatively younger male G. austeni population than that of G. pallidipes. The mean age of male G. austeni was estimated at 18 days, while that of G. pallidipes was estimated at 30 days. In contrast, ovarian ageing indicated that females of both species had comparable longevity and there was no significant difference between the frequency distribution of females among the eight ovarian categories (Table 1).

\section{Wolbachia and Sodalis infection prevalence in the two tsetse species}

The quality of template DNA, in particular the saltextracted samples, was confirmed by the $12 \mathrm{~S}$ assay as described above and negative samples excluded from 
Table 1 Frequency distribution (\%) of the ovarian categories in female G. austeni and G. pallidipes as determined by ovarian ageing

\begin{tabular}{|c|c|c|c|c|}
\hline \multirow{2}{*}{$\begin{array}{l}\text { Ovarian } \\
\text { category }\end{array}$} & \multirow{2}{*}{$\begin{array}{c}\text { Estimated age } \\
\text { (days) }\end{array}$} & \multicolumn{2}{|c|}{ Frequency (\%) } & \multirow[t]{2}{*}{ P-value } \\
\hline & & $\begin{array}{l}\text { G. pallidipes } \\
(\mathrm{n}=103)\end{array}$ & $\begin{array}{c}\text { G. austeni } \\
(n=67)\end{array}$ & \\
\hline 0 & $0-8$ & 1.6 & 4.5 & 0.6812 \\
\hline 1 & 8-19 & 7.8 & 14.9 & 0.2011 \\
\hline 2 & $20-30$ & 30.1 & 25.4 & 0.6016 \\
\hline 3 & $30-40$ & 22.3 & 22.4 & 1.000 \\
\hline 4 & $40-50$ & 13.6 & 16.4 & 0.6607 \\
\hline 5 & $50-60$ & 5.8 & 3.0 & 0.4820 \\
\hline 6 & $60-70$ & 12.6 & 10.4 & 0.8090 \\
\hline 7 & $70-80$ & 4.9 & 3.0 & 0.7052 \\
\hline
\end{tabular}

There were no significant differences in number of females per ovarian category between the two species.

further analysis. Subsequently, 370 G. austeni comprising 206 females and 164 males were analyzed for Wolbachia infection prevalence. Of these, 100 samples were collected from ASF, 196 from SHNR and the remaining 74 originated from South Africa. All 370 samples were Wolbachia-positive using the wsp gene. The presence of Wolbachia infections was confirmed in a subset of these samples $(n=50)$ using the $16 \mathrm{~S}$ rRNA-based PCR assay, and all samples were positive. Using this assay, only one amplification product of $400 \mathrm{bp}$ was observed. This is in contrast to the profile obtained for G. m. morsitans, where an additional band of $\sim 300 \mathrm{bp}$ is observed. In the organ-specific assay, Wolbachia was detected all body sections assayed including head, thorax, abdomen and legs (Figure 2a). Comparing the Kenyan and the South African populations, the intensity of the PCR amplification products from the latter population was consistently weaker than those of the former population, despite using the same volume of total genomic of DNA as PCR template (Figure 2b).
Out of 161 female and 141 male G. pallidipes from SHNR analysed, none was positive for Wolbachia.

The prevalence of Sodalis infections in G. austeni was $2 \%(n=100)$ in ASF and $4.6 \%(n=196)$ in SHNR as detailed in Table 2 . The difference in prevalence was not significant $(\mathrm{P}=0.3443)$ between the two areas. In the South African samples, the prevalence in LSLSA samples was $70.8 \%(n=24)$, while only one out of 50 flies analysed from the "northern" population was Sodalis positive. The prevalence of Sodalis was significantly different inter-country $(\mathrm{p}<0.0001)$. In the case of G. pallidipes, $16 \%(n=302)$ were infected with Sodalis (Table 2). Infection was biased towards females in G. austeni $(\mathrm{p}<0.0001)$, but no significant sex difference in infection was observed in $G$. pallidipes $(\mathrm{P}=0.0577)$.

\section{Temporal variation of Sodalis prevalence}

There was no statistical difference between Sodalis infection in ASF and SHNR and therefore the data were pooled to compare the temporal variance in prevalence. The total mean\% prevalence for Sodalis infection for the 3-year period was significantly higher in G. pallidipes than in G. austeni $(p<0.0001)$. There was no significant difference between the two species of flies in the year 2009 ( $\mathrm{P}=0.8014)$. However, as shown in Table 3, in 2010 and 2011 the prevalence was significantly higher in $G$. pallidipes than in G. austeni ( $\mathrm{p}<0.0001$, Fisher's exact test).

\section{Trypanosome infection in G. austeni and G. pallidipes}

Dissection of 787 flies comprising 235 G. austeni and 552 G. pallidipes yielded infection rates of $6 \%$ and $4.9 \%$ respectively. PCR analysis detected 71 trypanosome infections with various trypanosomes from 598 and 74 flies from Kenya and South Africa respectively (Figure 3). In the Kenyan G. austeni population, trypanosome prevalence in ASF was $13 \%(n=100)$ while in SHNR, it was $12.7 \%(n=196)$. However, the difference in prevalence was not significant $(\mathrm{P}=1.0000)$. In the South African population, no trypanosomes were detected in the LSLSA

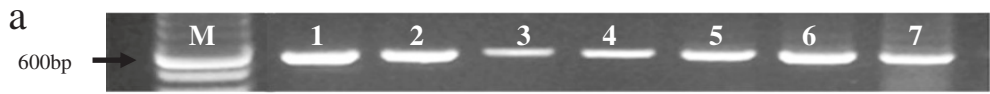

b

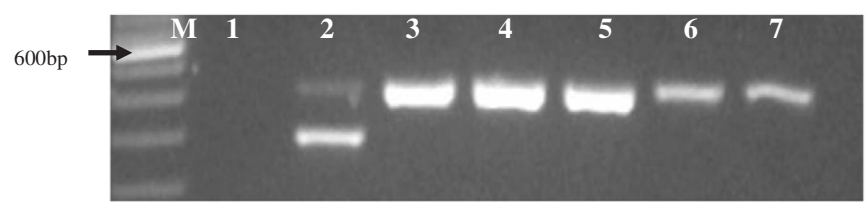

Figure 2 PCR analysis of Wolbachia organ distribution and comparative inter-country density. (a) Distribution of Wolbachia in G. austeni

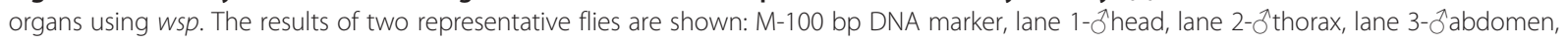
lane 4-9reproductive tract, lane 5-9head, lane 6-9thorax, lane 7-9legs. Arrow indicates 600 bp. (b) 16S rRNA analysis of G. austeni from Kenya and South Africa: M-100 bpDNA marker, Lane 1-NC, Lane 2-G. morsitans, Lanes 3, 4, 5-G. austeni Kenya, Lanes 6, 7-G. austeni South Africa. Arrow indicates $600 \mathrm{bp}$. Results of five representative samples are shown here out of 50 samples analyzed. 
Table 2 Sodalis prevalence in G. austeni and G. pallidipes from different locations

\begin{tabular}{lccccc}
\hline Species & Source & $\boldsymbol{n}$ & \multicolumn{2}{c}{ Number infected } & Prevalence \\
\cline { 3 - 4 } & & & $\mathbf{F}$ & $\mathbf{M}$ & \\
\hline \multirow{3}{*}{ G. austeni } & ASF, Kenya & 100 & $2 \%(0.00243 \leq \mathrm{p} \leq 0.07038)$ \\
& SHNR, Kenya & 196 & 6 & 3 & $4.6 \%(0.02121 \leq \mathrm{p} \leq 0.08538)$ \\
& St. Lucia, S. Africa & 24 & 17 & 0 & $70.8 \%(0.48905 \leq \mathrm{p} \leq 0.87385)$ \\
G. pallidipes & northern, S. Africa & 50 & 1 & 0 & $2 \%(0.00051 \leq \mathrm{p} \leq 0.10647$ \\
\hline
\end{tabular}

"northern" refers to sites north of LSLSA. F-females, M-males; prevalence \% given with $95 \%$ confidence intervals in brackets.

population, while a prevalence of $8 \%$ was detected in the northern population. An infection prevalence of 9.6\% $(\mathrm{n}=302)$ was observed in G. pallidipes. Combining all the species- and country-specific data, the total trypanosome prevalence was significantly higher in the Kenyan samples $(P=0.0425)$, but no significant difference was observed between the two fly species $(P=0.2435)$. In both tsetse species, proportionately more females than males were infected. This difference was significant in $G$. pallidipes $(\mathrm{p}<0.0001)$ but not in $\mathrm{G}$. austeni $(\mathrm{P}=0.8694)$. A temporal effect was evident with both species having a higher parasite infection rate in 2010 (Figure 4). Chisquare test showed that the differences in prevalence rates for the three years was significant in both G. austeni $\left(X^{2}=6.812, \mathrm{df}=2, \mathrm{P}=0.00332\right)$ and $\mathrm{G}$. pallidipes $\left(\mathrm{X}^{2}=2.321\right.$, $\mathrm{df}=2, \mathrm{P}=0.3133)$. ITS1 primers identified that several species of trypanosomes infect tsetse flies $(n=71)$ in the study area (Figure 4), the most common of which were T. congolense (46.5\%), T. vivax (38\%) and T. simiae (14.1\%). A small proportion of flies $(2.8 \%)$ had mixed infections of T. b. brucei and T. congolense (Table 4).

\section{Co-infection of Sodalis and trypanosomes}

Only a small proportion of flies sampled harboured both trypanosomes and Sodalis. Of these 3.3\% $(\mathrm{n}=302)$ and $1 \%(\mathrm{n}=296)$ were found in G. pallidipes and G. austeni, respectively. Flies that were microscopically-positive for trypanosomes were not always infected with Sodalis bacteria. The association between trypanosomes and Sodalis infection was statistically significant in G. pallidipes, but not in G. austeni (Table 5). Of the 13 co-infected flies, 5 were infected with $T$. congolense, 6 with $T$. simiae and 2 with $T$. vivax.

Table 3 Temporal variation of \% Sodalis infection prevalence in Kenyan G. austeni and G. pallidipes

\begin{tabular}{lllll}
\hline & \multicolumn{3}{c}{ Year } & \\
\cline { 2 - 4 } & $\mathbf{2 0 0 9}$ & $\mathbf{2 0 1 0}$ & $\mathbf{2 0 1 1}$ & Total \\
\hline G. austeni & $8.6 \%(116)$ & $1.5 \%(66)$ & $0 \%(114)$ & $3.7 \%(296)$ \\
G. pallidipes & $7 \%(100)$ & $27 \%(100)$ & $13.7 \%(102)$ & $15.9 \%(302)$ \\
$p$-value & $p=0.8014(\mathrm{NS})$ & $p<0.0001$ & $p<0.0001$ & $p<0.0001$ \\
\hline
\end{tabular}

Numbers in brackets represent $n$. The difference in total infection prevalence between the two species was highly significant.

\section{Discussion}

\section{Fly catches}

Three species of tsetse flies were captured in SHNR in increasing order of G. brevipalpis, G. austeni and G. pallidipes. The mixed type of vegetation found in this area provides suitable habitat for these savannah-dwelling species. About $75 \%$ of the total catch was G. pallidipes, influenced in part by the sampling tools. The study used biconical and Ngu traps, which are more effective for the capture of G. pallidipes than of G. austeni [43]. Sticky mono-panel traps are recommended for sampling of G. austeni [31] and indeed in the present study, this trap design captured more G. austeni than the other two traps. However, trap comparison was not an objective of our study and we did not therefore perform idealised trap comparison experiments. Flies caught on the sticky monopanel traps proved difficult to handle thereafter during dissection or DNA extraction due to the adhesive used on the panels and thus this trap was not routinely used. No G. pallidipes flies were captured in Arabuko-Sokoke forest. This can be attributed to the observation that G. pallidipes prefers open savannah grassland habitats, whereas the ASF habitat is composed mainly of dense indigenous forest.

\section{Prevalence of Wolbachia in G. austeni and G. pallidipes}

All G. austeni individuals analyzed were shown to be infected with Wolbachia. This result indicates an increase in infection prevalence towards complete fixation of Wolbachia considering that available 1996 data reported a prevalence of $48 \%$ in Shimba Hills and $98 \%$ in South Africa, respectively [28]. Population genetics models using data derived from G. m. morsitans predict that it would take about 18 months for Wolbachia prevalence to reach $95 \%$ of the fixation prevalence starting with a release ratio of $10 \%$ Wolbachia infected flies [21]. In relation to vector control, Wolbachia-mediated cytoplasmic incompatibility $(\mathrm{CI})$ is often proposed as a possible control intervention [44] and recently, unidirectional CI has been demonstrated to occur in a G. morsitans laboratory line [21]. Although the presence of $\mathrm{CI}$ in G. austeni remains to be confirmed, the high infection prevalence observed in this study indicates that 


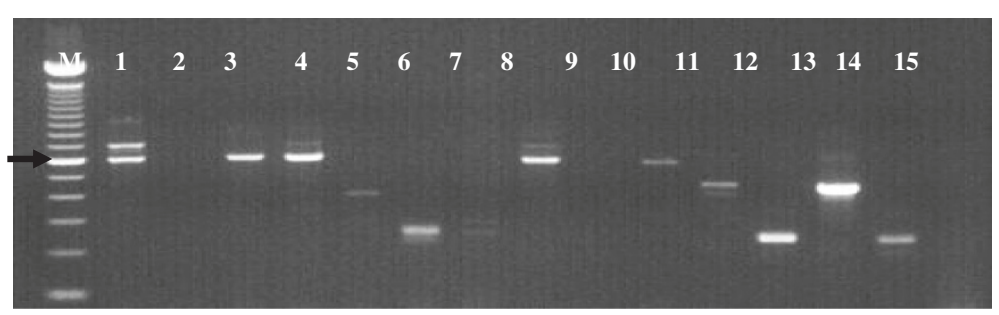

Figure 3 Identification of trypanosomes infecting tsetse flies using ITS1. Identification is based on product size. M-100 bp DNA marker: lanes 1, 3, 4, 8, 10-T. congolense sub-types, Lane 5-T. simiae: lanes 6, 7-T. vivax: lane 11-T. brucei: lane 13-positive control T. brucei: lanes 12, 14-positive controls for T. vivax. Lane 15-negative control. Arrow indicates $600 \mathrm{bp}$.

strong CI may be operating in this species as well. Our future work will confirm this by cross-mating natural populations with heterogeneous Wolbachia infection or by using tetracycline-cured laboratory lines.

The Wolbachia 16S rRNA analysis revealed only one band in G. austeni, unlike in G. morsitans, which displayed two distinct bands under the same amplification conditions. In the latter species, the larger product $(\sim 00 \mathrm{bp})$ represents the cytoplasmic product located in the reproductive organs, and which can be eliminated by tetracycline treatment. The smaller $300 \mathrm{bp}$ product represents either the Wolbachia infection found in various tissues or is a degenerate 16S rRNA copy suggestive of the lateral transfer of this gene from Wolbachia into G. morsitans [36]. Our results augment the available information highlighting the differences between Wolbachia strains in these two species and which has been confirmed by MLST analysis [36]. Our findings based on the Wolbachia 16S rRNA gene do not however, unequivocally confirm the presence or absence of an LGT event in G. austeni. It is therefore possible that the Wolbachia fixation we observed in G. austeni may be due to a LGT event, rather than an authentic Wolbachia infection. While it would be possible to confirm this using tetracycline-treated laboratory lines, this facility was not available to us during the period of the study. Additional work is therefore required to ascertain the presence of Wolbachia in aposymbiotic G. austeni.

Wolbachia density was greater in the Kenyan samples than in the South African samples based on the intensity of the PCR amplification product. The bacterial density and subsequently, the strength of $\mathrm{CI}$ expression may be influenced by the infecting Wolbachia strains. [45] However, since Wolbachia strains infecting the Kenyan and the South African tsetse populations are virtually identical [36], strain differences do not adequately address this discrepancy. Average Wolbachia densities may also vary between populations of the same species as a consequence of environmental factors [46], and it is possible that the density difference observed in the present study is population-specific. Wolbachia density levels in the different populations representing the G. austeni belt stretching many kilometers along the East African coast, would be of interest to explore. Also of interest would be the host genetic differentiation and the presence and levels of $\mathrm{CI}$ expression in these populations.

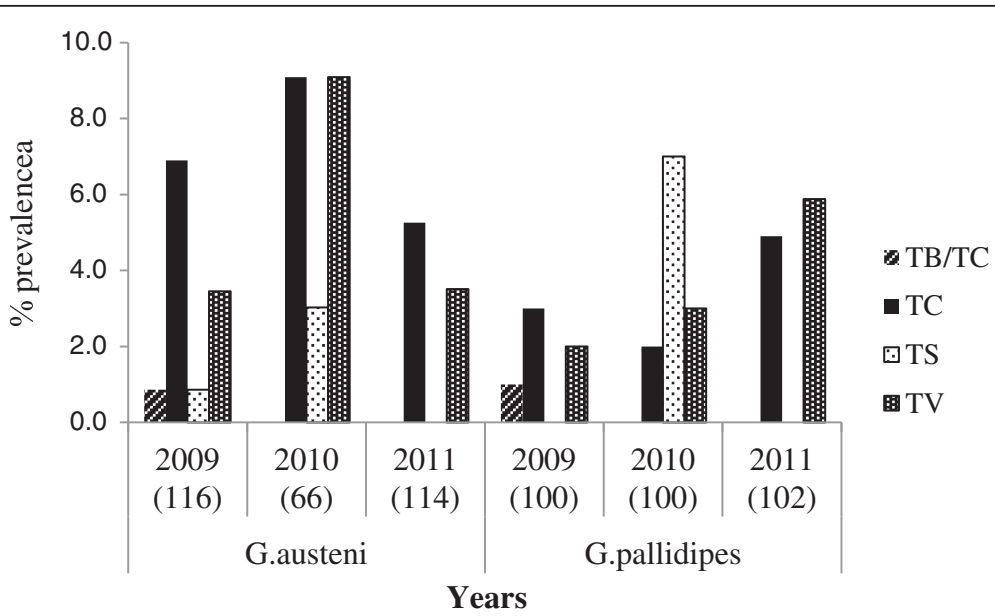

Figure 4 Trypanosome species infecting G. austeni and G. pallidipes for the period 2009-2011 as determined by PCR. Numbers in brackets represent total number of samples analysed for that specific year. TV-T. vivax, TS-T. simiae, TC-T. congolense, TB/TC-T. b. brucei/T. congolense mixed infection. 
Table 4 Trypanosome species detected in G. pallidipes and G. austeni. Trypanosome species detected in G. pallidipes and G. austeni

\begin{tabular}{|c|c|c|c|c|c|c|c|}
\hline Species & Source & $n$ & T.c. & T.v. & T.sm. & $T b b / T c$ & Total \\
\hline \multirow[t]{4}{*}{ G. austeni } & ASF, Kenya & 100 & 8 & 3 & 1 & 1 & $13(13 \%)$ \\
\hline & SHNR, Kenya & 196 & 12 & 11 & 2 & 0 & $25(12.7 \%)$ \\
\hline & St. Lucia, S. Africa & 24 & 0 & 0 & 0 & 0 & $0 / 24(0 \%)$ \\
\hline & northern, S. Africa & 50 & 2 & 2 & 0 & 0 & $4 / 50(8 \%)$ \\
\hline G. pallidipes & SHNR, Kenya & 302 & 11 & 11 & 7 & 1 & $29(9.6 \%)$ \\
\hline
\end{tabular}

T.c.-T. congolense, T.v.-T. vivax, T. sm.-T. simiae, T.b.b.-T. b. brucei. "northern" refers to sites north of LSLSA.

No Wolbachia infection was found in the G. pallidipes populations analyzed. This result is consistent with previously reported zero infection rates in Kenyan and Ugandan G. pallidipes populations [28]. Recently, however, a prevalence of $1.2 \%$ was reported from 1,823 samples originating from Ethiopia, Tanzania, Zambia and Zimbabwe [36]. It is likely that G. pallidipes Wolbachia infection is either absent or of too low a density to be detected using the standard PCR assay. Wolbachia infection in G. $f$. fuscipes which were undetectable by standard wsp PCR analysis were subsequently detected using Southern blot PCR technique $[47,48]$ and sequential amplifications [9]. The density of Wolbachia may play a role in expression of CI [49], thus the physiological significance of low-density Wolbachia infections in nature needs to be analyzed. G. pallidipes is a major vector for both human and animal trypanosomiasis in Kenya, and has been the target species in previous control programmes established in Nguruman, Busia and Lambwe Valley. For Wolbachiabased control strategies to be applied, a method of introducing the bacteria into this species would have to be developed. Tsetse are viviparous and the developing larvae acquire Wolbachia through germ-line transmission. Although methods for propagating Wolbachia in vitro are available [50], it may be difficult to introduce Wolbachia into the developing eggs because the embryonic microinjection technique used in other arthropods [51] cannot be easily applied in tsetse. The difficulty of mass-rearing this species [52], in order for the establishment of a sufficiently large self-sustaining laboratory colony of Wolbachia-infected G. pallidipes for large scale intervention would need to be overcome.

Table 5 Sodalis and trypanosome co-infection in field collected G. pallidipes and G. austeni

\begin{tabular}{|c|c|c|c|c|}
\hline & \multicolumn{2}{|c|}{ G. pallidipes $(n=302)$} & \multicolumn{2}{|c|}{ G. austeni $(n=296)$} \\
\hline & $\mathrm{T}+$ & T- & $\mathrm{T}+$ & T- \\
\hline$S+$ & $10(3.3 \%)$ & $38(12.6 \%)$ & $3(1.0 \%)$ & $8(2.7 \%)$ \\
\hline S- & $19(6.3 \%)$ & $235(77.8 \%)$ & $35(11.8 \%)$ & $250(84.5 \%)$ \\
\hline$P$-value & \multicolumn{2}{|c|}{$P=0.0127 *$} & \multicolumn{2}{|c|}{$P=0.1554(N S)$} \\
\hline
\end{tabular}

* significant, NS-not significant, $\mathrm{S}^{+} / \mathrm{S}^{-}$Sodalis positive/negative, $\mathrm{T}^{+} / \mathrm{T}$ trypanosome positive/negative.

\section{Prevalence of Sodalis in G. austeni and G. pallidipes}

Most commensal symbionts are considered non-essential and they are generally found at intermediate frequencies within host populations [53]. Sodalis was detected in Kenyan G. austeni and G. pallidipes at 3.7\% $(\mathrm{n}=296)$ and $15.9 \%(n=302)$ prevalence respectively. In contrast, Sodalis prevalence in the LSLSA G. austeni population was $70.5 \%$. This difference could be occasioned by environmental factors, which have been shown to influence symbiont densities in laboratory experiments [54]. In the present study, higher prevalence was obtained in fly samples from trap sites that are closer to the coastline (LSLSA) than those further inland, which may be attributed to marine-induced differences in the micro-climate. Sodalis infection prevalence also showed variation among the different species, with higher Sodalis infections in G. pallidipes. Among the riverine species, although G. $f$. fuscipes in the Lake Victoria, region apparently lack Sodalis infections $[8,9]$, the prevalence in west African G. $p$. palpalis has been reported to range from $9.3 \%$ to $54.9 \%[10,37,55]$.

\section{Temporal variation of Sodalis infection in G. pallidipes and G. austeni}

In the present study, the prevalence of Sodalis infection in both species more than doubled in 2010 as compared to the previous year. Temporal variations in symbiont infection prevalence may be correlated with climatic and ecological factors. In weevils, higher infections with Sodalisallied symbiont (Sitophilus oryzae primary endosymbiont (SOPE), Wolbachia and Rickettsia have been reported at localities of higher temperature [56]. Weather data from the Kenya coast indicates that the year 2010 was characterised by a poor short rainy season (October-December) when the depressed rainfall was coupled with higher temperatures as the dry weather conditions extended into the traditional short rainy season [57]. The samples analysed in this study were collected during the months of OctoberNovember when hotter, drier conditions prevailed. In contrast both 2009 and 2011 were characterized by nearnormal weather conditions. We postulate that environmental factors may have had an effect on the Sodalis prevalence in G. austeni and G. pallidipes 
Age structure of the sympatric G. austeni and G. pallidipes G. austeni males in the SHNR have a predicted shorter lifespan than the sympatric G. pallidipes, whereas the longevity of females is comparable in the two species. Some strains of Wolbachia have a life-shortening effect on their hosts. In a laboratory study, the wMelPop-CLA strain was shown to halve the lifespan of $A$. aegypti [22]. It has been hypothesized that this strain may be similar to that found in G. austeni [58]. Against this background, and considering the absence of Wolbachia in the sympatric G. pallidipes population, it could be possible that Wolbachia infection could be one of the factors contributing to shorter lifespan in G. austeni. However, the observation of this phenomenon in only male G. austeni may not be fully explained by this hypothesis. Future research will work towards confirming this finding in the field.

\section{Trypanosome infection in tsetse flies}

The observed trypanosome infection rates of $12.8 \%$ and $9.6 \%$ for G. austeni and G. pallidipes respectively are comparable with reported infection rates of $8.7 \%$ in G. pallidipes from the same area [59]. Our finding of $T$. congolense as the most common trypanosome circulating in this area is also consistent with previous reports of high cattle infection rates (upto 18\%), the majority of which were $T$. congolense infections [60]. Trypanosome infection prevalence was consistently higher in G. austeni than in G. pallidipes. It has been noted that despite the lower apparent density of $G$. austeni relative to other sympatric species, G. austeni often harbours higher levels of trypanosome infection [61]. The results obtained in the present study are in contrast to those obtained from Rufiji district along the Tanzanian coast whereby G. pallidipes was found to have a much higher infection prevalence than G. austeni [62]. Notably, more than half of the flies analyzed from the Rufiji population were infected with between two to five trypanosomes species while in this study, mixed infections accounted for only $2.8 \%$ of all infections. It is inferred that the presence of a wide spectrum of potential tsetse hosts, as well as sequential feeding upon multiple hosts may contribute to an abundance of mixed trypanosome infections [63]. However, since both of these regions have a wide variety of wild animals, the difference in prevalence of mixed infections may be a reflection of fly immunity, parasite maturation factors and possibly varying microfauna [64].

\section{Co-infection with Sodalis and trypanosomes}

Sodalis is transmitted through the milk gland secretions of the mother to the intra-uterine developing larva [65], and is therefore present in the gut at eclosion in the newly emerged teneral fly. On the other hand, trypanosome infection is acquired later in the presence of an infected blood meal source. The establishment of trypanosomes involves complex interactions and various factors including lectin levels in the tsetse fly gut at the time of parasite uptake, fly species, sex, age and symbiotic associations in the tsetse fly gut play a part in determining the success or failure of parasite establishment [2]. Midgut symbionts such as Sodalis may exhibit a chitinolytic activity [66] which increases the susceptibility of tsetse fly to trypanosome infection [11]. Of the almost 600 tsetse samples that we analyzed, less than $2 \%$ were found coinfected with both Sodalis and trypanosomes. This result is not surprising given that very few flies were positive for any of the two organisms. Only ten G. pallidipes and 3 G. austeni were co-infected with both microorganisms. Therefore, despite positive correlation between presence of Sodalis and midgut trypanosome infections in natural populations [11,67], it is clear that tsetse flies can become infected with trypanosomes readily in the absence of Sodalis infections both in the laboratory and in the field $[7,68]$. The low co-infection rates observed in the present study are in contrast with the rates of $32.2 \%$ reported in G. p. palpalis [10]. It is, however, worth noting that in both the present study and the G. p. palpalis study, the trypanosomes species infecting Sodalis-positive flies belong mainly to the sub-groups Trypanozoon and Nannomonas. Intercommunity effects have been observed between symbionts and pathogens, where a negative correlation between Wolbachia and trypanosomes and a positive correlation between the salivary gland hypertrophy (SGH) virus and trypanosomes were noted in Ugandan G. $f$. fuscipes [9]. Thus, it remains to be seen if the apparent absence of Wolbachia infections in G. pallidipes may render these populations more susceptible to host Sodalis and/or trypanosome infections. Further, more work is required to determine whether the synergistic effect of Sodalis on trypanosome establishment and maturation may apply favorably to trypanosome species that particularly involve the tsetse midgut stages [69]. However, in this study the number of co-infected flies was too low to provide a strong argument for the antithesis. Further work on this aspect will serve to provide conclusive evidence.

\section{Conclusion}

In summary, this study found that the natural prevalence of Sodalis and Wolbachia symbionts in G. pallidipes and G. austeni in coastal Kenya differ between the two species. The apparently absent or possibly low-titer infections observed in G. pallidipes complicate the feasibility for application of a Wolbachia-mediated CI control approach against this species. However, IIT could be possible for G. austeni if the CI effect is confirmed in this species. In the populations analysed in East Africa, there is low level of natural co-infection with the secondary endosymbiont and trypanosomes and this could indicate 
limited influence of Sodalis on trypanosome infection. Proposed symbiont-mediated control interventions should take these differences into account and particularly the low levels of infection.

\section{Competing interests}

The authors declare that they have no competing interests.

\section{Authors' contributions}

FNW, RMN, SA conceived the experiments, FNW, PTC collected the field samples, JOO, SA contributed reagents, FNW, UA, EA carried out the analysis, FNW, SA, UA wrote the manuscript. All authors read and approved the final version of the manuscript.

\section{Acknowledgements}

This work was supported by National Council for Science and Technology PhD grant to FNW, WHO/TDR A80132 to JOO and NIH Fogarty awards D43TW007391 and R03TW008413 to SA. South African samples graciously provided by Otto Koekemoer $(\mathrm{OVI})$. We thank the Kenya Wildlife Service (KWS) for granting us access into the wildlife parks, TRC Entomology Division staff for technical assistance, the Director KARI and the Centre Director, TRC for logistical support and Paul Mireji for useful comments on the manuscript.

\section{Author details}

'KARI-Trypanosomiasis Research Centre, P.O. Box 362-00902, Kikuyu, Kenya. ${ }^{2}$ Yale University, School of Public Health, 60 College Street, 811 LEPH, New Haven, CT 06520, USA. ${ }^{3}$ Department of Biochemistry and Molecular Biology, Egerton University, P.O. Box 536-20115, Egerton, Kenya.

Received: 6 May 2013 Accepted: 31 July 2013

Published: 8 August 2013

\section{References}

1. Barrett $\mathrm{K}$, Okali C: Partnerships for tsetse control: community participation and other options. World Anim Rev 1998, 90:39-46.

2. Welburn SC, Maudlin I: Tsetse-typanosome interactions: rites of passage. Parasitol Today 1999, 15:399-403.

3. Aksoy S, Gibson WC, Lehane MJ: Interactions between tsetse and trypanosomes with implications for the control of trypanosomiasis. Adv Parasitol 2003, 53:1-83.

4. Hao Z, Kasumba I, Lehane MJ, Gibson WC, Kwon J, Aksoy S: Tsetse immune responses and trypanosome transmission: implications for the development of tsetse-based strategies to reduce trypanosomiasis. Proc Natl Acad Sci U S A 2001, 98(22):12648-12653.

5. Nogge G: Sterility in tsetse flies Glossina morsitans Westwood caused by loss of symbionts. Experientia 1976, 32:995-996.

6. Wang J, Wu Y, Yang G, Aksoy S: Interactions between mutualist Wigglesworthia and tsetse peptidoglycan recognition protein (PGRP-LB) influence trypanosome transmission. Proc Natl Acad Sci USA 2009, 106(29):12133-12138.

7. Pais R, Lohs C, Wu Y, Wang J, Aksoy S: The Obligate Mutualist Wigglesworthia glossinidia influences reproduction, digestion, and immunity processes of its host, the tsetse fly. Appl Environ Microbiol 2008, 74:5965-5974.

8. Lindh JM, Lehane MJ: The tsetse fly Glossina fuscipes fuscipes (Diptera: Glossina) harbours a surprising diversity of bacteria other than symbionts. Antonie Leeuwenhoek 2011, 99:711-720.

9. Alam U, Hyseni C, Symula RE, Brelsfoard C, Wu Y, Kruglov O, Wang J, Echodu R, Alioni V, Okedi LM, Caccone A, Aksoy S: Microfauna-host interactions: implications for trypanosome transmission dynamics in Glossina fuscipes fuscipes in Uganda. Appli Environ Microbiol 2012, 78(13):4627-4637.

10. Farikou O, Njiokou F, Mbida JAM, Njitchouang GR, Djeunga HN, Asonganyi T, Simarro PP, Cuny G, Geiger A: Tripartite interactions between tsetse flies, Sodalis glossinidius and trypanosomes-An epidemiological approach in two historical human African trypanosomiasis foci in Cameroon. Infect Genet Evol 2010, 10:115-121.

11. Geiger A, Ravel S, Mateille T, Janelle J, Patrel D, Cuny G, Frutos R: Vector competence of Glossina palpalis gambiensis for Trypanosoma brucei s.l. and genetic diversity of the symbiont Sodalis glossinidius. Mol Biol Evol 2007, 24:102-109.

12. Weiss $B$, Aksoy S: Microbiome influences on insect host vector competence. Trends Parasitol 2011, 27(11):514-522.

13. Weiss BL, Maltz M, Aksoy S: Obligate symbionts activate immune system development in the tsetse fly. J Immunol 2012, 188(7):3395-3403.

14. Beard CB, Cordon-Rosales C, Durvasula RV: Bacterial symbionts of the Triatomine and their potential use in control of Chagas disese transmission. Annu Rev Entomol 2002, 47:123-141.

15. Hurwitz I, Hillesland H, Fieck A, Das P, Durvasula R: The paratransgenic sand fly: a platform for control of Leishmania transmission. Parasit Vectors 2011, 19(4):82.

16. Riehle MA, Moreira CK, Lampe D, Lauzon C, Jacobs-Lorena M: Using bacteria to express and display anti-Plasmodium molecules in the mosquito midgut. Int J Parasitol 2007, 37:595-603.

17. Chavshin AR, Oshaghi MA, Vatandoost H, Pourmand MR, Raeisi A, Enayati AA, Mardani N, Ghoorchian S: Identification of bacterial microflora in the midgut of the larvae and adult of wild caught Anopheles stephensi: a step toward finding suitable paratransgenesis candidates. Acta Trop 2012, 121:129-134.

18. Rasgon JL: Using infections to fight infections: paratransgenic fungi can block malaria transmission in mosquitoes. Future Microbiol 2011, 6:851-853.

19. Laven H: Eradication of Culex pipiens fatigans through Cytoplasmic Incompatibility. Nature 1967, 216:383-384.

20. Zabalou S, Riegler M, Theodorakopoulou M, Savakis C, Bourtzis K: Wolbachia-induced cytoplasmic incompatibility as a means for insect pest population control. Proc Natl Acad Sci USA 2004, 101:15042-15045.

21. Alam U, Medlock J, Brelsfoard C, Pais R, Lohs C, Balmand S, Carnogursky J, Heddi A, Takac P, Galvani A, Aksoy S: Wolbachia symbiont infections induce strong cytoplasmic incompatibility in the tsetse fly Glossina morsitans. PLoS Pathog 2011, 7(12):e1002415.

22. Min KT, Benzer S: Wolbachia, normally a symbiont of Drosophila, can be virulent, causing degeneration and death. Proc Natl Acad Sci USA 1997, 94:10792-10796.

23. Cook PE, McMeniman CJ, O'Neill SL: Modifying insect population age structure to control vector-borne disease. Adv Exp Med Biol 2008, 627:126-140.

24. Moreira LA, Iturbe-Ormaetxe I, Jeffery JA, Lu G, Pyke AT, Hedges LM, Bruno R, Hall-Mendelin S, Day A, Riegler M, Hugo LE, Johnson KN, Kay BH, McGraw EA, van de Hurk AF, Ryan P, O'Neill SL: A Wolbachia symbiont in Aedes aegypti limits infection with dengue, Chikungunya and Plasmodium. Cell 2009, 139(7):1268-1278.

25. van den Hurk AF, Hall-Mendelin S, Pyke AT, Frentiu FD, McElroy K, Day A, Higgs S, O'Neill SL: Impact of Wolbachia on infection with chikungunya and yellow fever viruses in the mosquito vector Aedes aegypti. PLoS Negl Trop Dis 2012, 6:e1892.

26. Iturbe-Ormaetxe I, Walker T, Neill SLO: Wolbachia and the biological control of mosquito-borne disease. EMBO Rep 2011, 12(6):508-518.

27. Turelli M, Hoffmann AA: Microbe-induced cytoplasmic incompatibility as a mechanism for introducing transgenes into arthropods populations. Insect Biochem Mol Biol 1999, 8:243-255.

28. Cheng Q, Ruel TD, Zhou W, Moloo SK, Majiwa P, O'Neill SL, Aksoy S: Tissue distribution and prevalence of Wolbachia infections in tsetse flies, Glossina spp. Med Vet Entomol 2000, 14:44-50.

29. Challier A, Laveissiere C: Un nouveau piege pour la capture des glossines (Glossina: Diptera, Muscidae): description et essays sur le terrain. Cahiers ORSTOM, series Entomologie Medical Parasitologie 1973, 11:251-262.

30. Brightwell R, Dransfield RD, Kyorku C, Golder TK, Tarimo SA, Mungai D: A new trap for Glossina pallidipes. Trop Pest Manag 1987, 33:151-189.

31. Vreysen MJB, Saleh KM, Zhu Z-R, Suleiman FW: Responses of Glossina austeni to sticky panels and odours. Med Vet Entomol 2000, 14:283-289.

32. Torr SJ, Holloway MTP, Vale GA: Improved persistence of insecticide deposits on targets for controlling Glossina pallidipes (Diptera: Glossinidae). Bull Entomol Res 1992, 82:525-533.

33. Sunnucks $P$, Hales DF: Numerous transposed sequences of mitochondrial cytochrome oxidase I-II in aphids of the genus Sitobion (Hemiptera: Aphididae). Mol Biol Evol 1996, 13:510-524.

34. Braig HR, Zhou W, Dobson S, O'Neill S: Cloning and characterisation of a gene encoding the major surface protein of the bacterial endosymbiont Wolbachia pipientis. J Bacteriol 1998, 180:2373-2378. 
35. Zhou WG, Rousset F, O'Neill S: Phylogeny and PCR-based classification of Wolbachia strains using wsp gene sequences. Proc $R$ Soc Lond B Biol Sci 1998, 265:509-515

36. Doudoumis V, Tsiamis G, Wamwiri F, Brelsfoard C, Alam U, Aksoy $E_{1}$ Dalaperas S, Abd-Alla AMM, Ouma J, Takac P, Aksoy S, Bourtzis K: Detection and characterization of Wolbachia infections in laboratory and natural populations of different species of tsetse flies (genus Glossina). BMC Microbiol 2012, 12:S3.

37. Dale C, Maudlin I: Sodalis gen. nov. and Sodalis glossinidius sp. nov., a microaerophilic secondary endosymbiont of the tsetse fly Glossina morsitans morsitans. Int J Syst Bacteriol 1999, 49:267-275.

38. Simon C, Frati F, Beckenbach A, Crespi B, Liu H, Flook P: Evolution, weighting and phylogenetic utility of mitochondrial gene sequences and a compilation of conserved polymerase chain reaction primers. Ann Entomol Soc Am 1994, 87:651-704.

39. Lloyd L, Johnson WB: The trypanosome infections of tsetse flies in Northern Nigeria and a new method of estimation. Bull Entomol Res 1924, 14:265-288

40. Njiru ZK, Constantine CC, Guya S, Crowther J, Kiragu JM, Thompson RC, Dávila AM: The use of ITS1 rDNA PCR in detecting pathogenic African trypanosomes. Parasitol Res 2005, 95(3):186-192.

41. Jackson CHN: An artificially isolated generation of tsetse flies (Diptera). Bull Entomol Res 1946, 37:291-299.

42. Saunders DS: Age determination for female tsetse flies and the age compositions of samples of Glossina pallidipes Austen, G. palpalis fuscipes Newst. and G.brevipalpis Newst. Bull Entomol Res 1962, 53:579-595.

43. Takken W: Studies on the biconical trap as a sampling device for tsetse (Diptera: Glossinidae) in Mozambique. Int J Trop Insect Sci 1984, 5:357-361.

44. Brelsfoard CL, Dobson SL: An update on the utility of Wolbachia for controlling insect vectors and disease transmission. AsPac J Mol Biol Biotechnol 2011, 19(3):85-92.

45. Clancy DJ, Hoffmann AA: Environmental effects on cytoplasmic incompatibility and bacterial load in Wolbachia-infected Drosophila simulans. Entomol Exp Appl 1998, 86:13-24.

46. Mouton L, Henri H, Charif D, Boulétreau M, Vavre F: Interaction between host genotype and environmental conditions affects bacterial density in Wolbachia symbiosis. Biol Lett 2007, 3(2):210-213.

47. Arthofer W, Riegler M, Schneider D, Krammer M, Miller WJ: Hiden Wolbachia diversity in field populations of the European cherry fruit fly Rhagoletis cerasi (Diptera, Tephritidae). Mol Ecol 2009, 18:3816-3830.

48. Miller WJ, Schneider D: Deciphering Wolbachia diversity in Glossina spp. In Improving SIT for tsetse flies through research on their symbionts and pathogens: 2010. Nairobi, Kenya: IAEA; 2010.

49. Ikeda T, Ishikawa H, Sasaki T: Infection density of Wolbachia and level of cytoplasmic incompatibility in the Mediterranean flour moth, Ephestia kuehniella. J Invertebr Pathol 2003, 84:1-5.

50. O'Neill S, Pettigrew M, Sinkins S, Braig HR, Andreadis T, Tesh R: In vitro cultivation of Wolbacha pipientis in an Aedes albopictus cell line. Insect Mol Biol 1997, 6:33-39.

51. McMeniman CJ, Lane RV, Cass BN, Fong AW, Sidhu M, Wang YF, O'Neill SL: Stable introduction of a life-shortening Wolbachia infection into the mosquito Aedes aegypti. Science 2009, 323:141-144.

52. Abd-Alla AMM, Parker AG, Vreysen MJB, Bergoin M: Tsetse salivary gland hypertrophy virus: hope or hindrance for tsetse control? PLOS Negl Trop Dis 2011, 5(8):e1220

53. Russel JA, Moran NA: Costs and Benefits of symbiont infection in aphids: variation among symbionts and across temperatures. Proc $R$ Soc Lond 2006, 273:603-610.

54. Rio RV, Wu YN, Filardo G, Aksoy S: Dynamics of multiple symbiont density regulation during host development: tsetse fly and its microbial flora. Proc R Soc Lond B Biol Sci 2006, 273:805-814

55. Maudlin I, Welburn SC, Mehlitz D: The relationship between rickettsia-like organisms and trypanosome infections in natural populations of tsetse in Liberia. Trop Med Parasitol 1990, 41:265-267.

56. Toju H, Fukatsu T: Diversity and infection prevalence of endosymbionts in natural populations of the chestnut weevil: relevance of local climate and host plants. Mol Ecol 2011, 20(4):853-868

57. GoK: The 2010 Short Rains Season Assessment Report. Nairobi: Government of Kenya; 2011:40.

58. Aksoy S: Tsetse: a haven for microorganisms. Parasitol Today 2000, 16:114-118.
59. Tarimo SA, Snow WF, Butler L: Trypanosome infections in wild tsetse, Glossina pallidipes Austen on the Kenya coast. Int J Trop Insect Sci 1984 5(05):415-418

60. Ohaga SO, Kokwaro ED, Ndiege IO, Hassanali A, Saini RK: Livestock farmers' perception and epidemiology of bovine trypanosomiasis in Kwale District, Kenya. Prev Vet Med 2007, 80:24-33.

61. Motloang M, Masumu J, Mans B, Van den Bossche P, Latif A: Vector competence of Glossina austeni and Glossina brevipalpis for Trypanosoma congolense in KwaZulu-Natal, South Africa. Onderstepoort J Vet Res 2012, 79(1):e1-e6.

62. Malele II, Magwisha HB, Nyingilili HS, Mamiro KA, Rukambile EJ: Multiple Trypanosoma infections are common amongst Glossina species in the new farming areas of Rufiji district, Tanzania. Parasit Vectors 2011, 4:217.

63. Simo G, Silatsa B, Njiokou F, Lutumba P, Mansinsa P, Madinga J, Manzambi $E$, De Deken $R$, Asonganyi T: Identification of different trypanosome species in the mid-guts of tsetse flies of the Malanga (Kimpese) sleeping sickness focus of the Democratic Republic of Congo. Parasit Vectors 2012, $5: 201$

64. Njiru ZK, Makumi JN, Okoth S, Ndungu JM, Gibson WC: Identification of trypanosomes in Glossina pallidipes and G. longipennis in Kenya. Infect Genet Evol 2003, 4:29-35.

65. Cheng Q, Aksoy S: Tissue tropism, transmission and expression of foreign genes in vivo in midgut symbionts of tsetse flies. Insect Mol Biol 1999, 8:125-132.

66. Ibrahim EAR, Ingram GA, Molyneux DH: Haemagglutinins and parasite agglutinins in heamolymph and gut of Glossina. Trop Med Parasitol 1984 35:151-156

67. Geiger A, Ravel S, Frutos R, Cuny G: Sodalis glossinidius (Enterobacteriaceae) and vectorial competence of Glossina palpalis gambiense and Glossina morsitans morsitans for Trypanosoma congolense savannah type. Curr Microbiol 2005, 51(1):35-40.

68. Weiss BL, Mouchotte R, Rio RV, Wu YN, Wu Z, Heddi A, Aksoy S: Interspecific transfer of bacterial endosymbionts between tsetse fly species: infection establishment and effect on host fitness. Appl Environ Microbiol 2006, 72:7013-7021.

69. Welburn SC, Arnold K, Maudlin I, Gooday GW: Rickettsia-like organisms and chitinase production in relation to transmission of trypanosomes by tsetse flies. Parasitol 1993, 107:141-145.

doi:10.1186/1756-3305-6-232

Cite this article as: Wamwiri et al:: Wolbachia, Sodalis and trypanosome co-infections in natural populations of Glossina austeni and Glossina pallidipes. Parasites \& Vectors 2013 6:232

\section{Submit your next manuscript to BioMed Central and take full advantage of:}

- Convenient online submission

- Thorough peer review

- No space constraints or color figure charges

- Immediate publication on acceptance

- Inclusion in PubMed, CAS, Scopus and Google Scholar

- Research which is freely available for redistribution 